\title{
Kants transzendentale Deduktion aus der Perspektive der Wissenschaft der Logik
}

Kant hat die kategorialen Grundbestimmungen der Gegenstände in der metaphysischen Deduktion als Begriffe des reinen Verstandes einzeln aus den logischen Funktionen „des Verstandes in Urteilen“ hergeleitet, um sie anschließend in der transzendentalen Deduktion en bloc als objektiv gültig zu legitimieren. Unser Hauptaugenmerk ruht auf der transzendentalen Deduktion, doch betrachten wir zuvor kurz die metaphysische.

\section{Die Herleitung der Kategorien aus der Syllogistik}

Aristoteles hatte gelehrt, dass die verschiedenen Prädikationen nicht erst kraft ihres Inhaltes dies und das bedeuten - Bäume, Pferde, Menschen, Weißes, Gebildetes u. s. w. - , sondern schon kraft ihrer bloßen Form. Die Formen der Prädikation, ta schêmata tês katêgorias, ${ }^{1}$ bezeichnen Grundbestimmungen des Seienden, eben die sogenannten Kategorien. Die Prädikationen „ist eine Eiche“, „ist ein Pferd“ und „ist ein Mensch“ etwa bezeichnen ihrem Inhalt nach verschiedene Substanzen und ihrer Form nach die Substanz als solche. Die Prädikationen „ist weiß“ und ,ist gebildet“ bezeichnen ihrem Inhalt nach verschiedene Qualitäten und ihrer Form nach die Qualität als solche, und so weiter für insgesamt zehn Kategorien, zehn logisch-grammatische Klassen von Prädikaten.

Aristoteles glaubte, man könne durch solches logisch-grammatische Botanisieren von Prädikaten verschiedene Grundbedeutungen des Wortes „sein“ freilegen, denen dann verschiedene höchste Gattungen von Seiendem entsprächen, und Carnap und Sellars haben sich ihm im vergangenen Jahrhundert insoweit angeschlossen, als auch sie die Kategorien als grundlegende metasprachliche Einteilungsprinzipien behandelten. Kant hingegen rügt das Botanisierende dieses Verfahrens: Aristoteles habe die Kategorien aufgerafft, „wie sie ihm aufstießen“2, und will ein neues, prinzipiengeleitetes Verfahren an die Stelle des alten setzen, sinnigerweise ein Verfahren, das Aristoteles selber bereitgestellt, von dem er aber für die Kategoriengewinnung keinen Gebrauch gemacht hatte. Aristoteles nämlich ist der Begründer der Syllogistik, die die Grundlage der traditionellen formalen Logik bildet und die auch durch die moderne Fregesche Prädikatenlogik nicht außer Kraft gesetzt, sondern in sie eingebettet wurde. An den Syllogismen lassen sich drei Geltungsmodi von Urteilen ablesen: Für die Prämissen wird im allgemeinen assertorische Geltung beansprucht, aber relativ zu ihnen soll die Konklusion dann apodiktisch gelten, und in hypothetischen und disjunktiven Prämissen kommen Teilurteile mit nur problematischer Geltung vor. So gibt die Syllogistik drei logisch relevante Modi der Geltung von Urteilen zu erkennen. Ferner kommen außer hypothetischen und disjunktiven Urteilen in Syllogismen kategorische Urteile vor, und diese unterscheiden sich durch die Quantität ihrer logischen Subjekte und die Qualität ihrer Prädikationen bzw. ihrer Kopulae in logisch relevanten Weisen. Daher lassen sich die Hinsichten der Modalität, Relation, Quantität und Qualität der Urteile mit ihren jeweils dreifachen Unterteilungen bequem an den Variationen des Syllogismus ablesen, und die kantische Tafel mit ihren vier mal drei logischen Funktionen in Urteilen ist nach Maßgabe der Syllogistik als des unbestrittenen Fundamentes der traditionellen formalen Logik zweifellos vollständig. (Ob durch die Fregesche

1 Aristoteles, Metaphysik D 7, 1017a23.

$2 \mathrm{KrV}$ A 81 / B 107. 
Prädikatenlogik neue Gesichtspunkte ins Spiel kommen, bleibt natürlich zu prüfen.) Hegel wäre übrigens der letzte, die Vollständigkeit der kantischen Urteilstafel zu bestreiten, macht er doch eigens - noch dazu mit recht erkünstelter Begründung - eine Ausnahme von seinem sonstigen Dreierschema, um Kants vierfache Einteilung der Urteile übernehmen und vertiefen zu können. Maß nehmend an Kants Unterscheidung von Qualität, Quantität, Relation und Modalität unterscheidet Hegel das Urteil der Qualität, der Reflexion, der Notwendigkeit und des Begriffs und entschuldigt seine Vierteilung damit, dass sie in Wahrheit eine Dreiteilung mit einem gespaltenen Mittelglied sei. Die Reflexion und die Notwendigkeit seien nämlich der mittleren logischen Sphäre, dem Wesen, zuzuordnen, welches die Sphäre der Dualität sei. Allerdings hatte dieser Sachverhalt Hegel keinesfalls dazu bewogen, auch die Wesenslogik selber in zwei Seiten aufzuspalten.

Halten wir also fest, dass die Unterscheidung von vier mal drei Urteilstypen zwischen Hegel und Kant nicht strittig ist. Kant legitimiert sie, zugegeben etwas äußerlich, aber durchaus hinreichend für die Zwecke einer quasijuristischen Deduktion, aus der Syllogistik. Hegel entwickelt umgekehrt die Syllogistik aus der Urteilslehre und das Urteil aus dem Begriff - ein Verfahren, das zu tieferen Einblicken in die Natur des Logischen führen mag (als Kant sie bietet). Aber Kant ist ökonomisch im Beweisen und greift deswegen auf das juristische Vorbild einer Deduktion zurück, in der nicht weniger und nicht mehr geklärt werden muss als nur das, was zwischen zwei Parteien strittig ist. Für diese reduzierten Beweisabsichten ist sein Verfahren vollkommen angemessen.

Wenn es um die Legitimität der Kategorien geht, sind die streitenden Parteien natürlich keine faktischen Positionen der Philosophiegeschichte, etwa der historische Rationalismus und der historische Empirismus, die vor dem Gerichtshof der Vernunft durch, sagen wir, ihre Advokaten Christian Wolff bzw. David Hume vertreten wären, sondern idealisierte Extremstandpunkte, nämlich ein rationaler Kategoriendogmatismus und ein radikaler Kategorienskeptizismus. Da zwischen diesen idealisierten Standpunkten die Syllogistik nicht strittig ist - so wenig wie de facto zwischen Wolff und Hume -, darf Kant sie seiner metaphysischen Deduktion als Ausgangspunkt zugrunde legen. Wie aber kommt er von den vier mal drei syllogistisch relevanten Urteilstypen zu ebenso vielen Grundbegriffen von Gegenständen? Nun, den Urteilstypen entsprechen Denkinhalte, allerdings keine Begriffsinhalte, sondern rein logische, formale Inhalte, die in der Sprache nicht durch Termini, sondern durch logische Partikeln wie „alle“, „einige“, die Kopula, die Negation, Junktoren und Modaloperatoren ausgedrückt werden. An dieser Stelle müssen wir uns an Kants Hume-Rezeption erinnern. Hume hatte zu Kants Zufriedenheit gezeigt, dass bestimmte fundamentale Begriffe, insbesondere der Begriff der Substanz und die Begriffe der Ursache und der Wirkung sich nicht empirisch legitimieren lassen. Wir denken in ihnen mehr, als wir je wahrnehmen können. Doch nach Hume hat dieses Mehr keine objektive Gültigkeit, sondern ist ein Produkt subjektiver Gewohnheit. So ist zum Beispiel die vermeintliche Notwendigkeit, mit der ein Ereignis qua Wirkung auf ein anderes Ereignis qua Ursache folgt, nur Ausdruck einer subjektiven Assoziation von Ideen aufgrund von Gewöhnung an beobachtete Abfolgen von Ereignissen.

In dieser Theorielage hat Kant außer von Hume auch von Aristoteles gelernt und beide Vorgänger in einem Zug überboten. Hume suchte nach der Rechtfertigung unseres Substanz- und Kausalitätsbegriffes in den Mustern unserer Sinneseindrücke und konnte dabei immerhin deutlich machen, dass hier nichts zu finden ist. Kant schloss daraus, dass Hume an der falschen Stelle gesucht habe; nicht in den Sinneseindrücken, sondern in der formalen Logik wäre er fündig geworden, denn der Begriff der Substanz ergibt sich mühelos aus der Form des kategorischen Urteils als der Begriff eines letzten Subjektes und der Begriff der Kausalität ebenso mühelos aus der Form des hypothetischen Urteils als der Begriff einer hinreichenden Bedingung in rebus nach Naturgesetzen. Nicht subjektive Gewöhnung an faktische Regelmäßigkeiten, sondern die formale Logik ist die Quelle unserer Substanz- und Kausalitätsbegriffe. Damit sind sie bereits in gewisser Hinsicht deduziert, d. h. vernünftig legitimiert.

In einem damit wird klar, dass man in der Kategorienlehre mit Aristoteles über Aristoteles hinausgehen muss. Denn wenn sich die Inhalte der beiden von Hume untersuchten Grundbegriffe aus der formalen Logik herleiten lassen, so sollten sich auf gleichem Wege weitere Grundbegriffe als Kategorien ausweisen lassen, nämlich insgesamt so viele, wie die Urteilstafel Unterschei- 
dungen zu machen erlaubt. Man braucht demnach nur die logisch relevanten Denkinhalte, die durch logische Partikeln (,alle“, „einige“, „ist“, „nicht“, „wenn ..., dann ...“, u. s. w.) ausgedrückt werden, in eine andere Inhaltsart zu transponieren, in die Art der Begriffsinhalte, und schon sind die Grundbegriffe des reinen Verstandes gewonnen und zugleich durch ihren logischen Ursprung als reine Verstandesbegriffe legitimiert.

\section{Die Frage der objektiven Gültigkeit der Kategorien}

Allerdings ist diese Legitimation noch nicht vollkommen. Denn auch wenn die Kategorien logische Inhalte in Form von Termini sind, so ist damit ihre objektive Gültigkeit noch nicht gesichert. Man denke zum Vergleich an die Problematik der Rechtfertigung unserer reinen Vernunftbegriffe - Freiheit, Seele, Gott - durch die praktische Vernunft. Kant kam hier zu dem Ergebnis, dass es zwar vollkommen rational sei, die Freiheit für real, die Seele für unsterblich und Gott für existierend zu halten, da dies Postulate der reinen praktischen Vernunft seien - aber eben doch bloß Postulate, also Setzungen. Gewiss handelt es sich um Setzungen mit Objektivitätsanspruch, aber mit einem Objektivitätsanspruch, der nicht unabhängig von der Setzung auszuweisen und zu legitimieren ist. Es fehlt für Setzungen der praktischen Vernunft grundsätzlich die Möglichkeit eines unabhängigen epistemischen Zugangs zum Gesetzten.

Dennoch sind diese Setzungen absolut rational. Man denke zum Vergleich an relativ rationale Setzungen, z. B. an David Lewis` Viele-Welten-Hypothese. Lewis postuliert die Welten um ihres erhofften Nutzens für philosophische Analysen willen, und er postuliert sie mit Objektivitätsanspruch als große konkrete Einzeldinge, die voneinander raumzeitlich und kausal isoliert sind. Wegen ihrer Isolation kann es keinen unabhängigen epistemischen Zugang zu anderen Welten geben; als physikalische Hypothese wäre der Weltenpluralismus daher eine Totgeburt. Für die Philosophie, sagt Lewis, ist er das Paradies. Allein deshalb also, weil eine prinzipiell nicht unabhängig legitimierbare Hypothese philosophische Analysen erleichtert, hält Lewis es für rational, sie mit Objektivitätsanspruch zu behaupten. Um wie vieles rationaler ist es dann, Voraussetzungen, die die reine Vernunft selber mit Objektivitätsanspruch um ihrer eigenen Konsistenz willen machen muss, d. h. die Postulate der reinen praktischen Vernunft, für zutreffend zu halten, in einem durch und durch vernünftigen Glauben, auch wenn sie ebenso wenig unabhängig belegt werden können wie die Lewis'schen Welten!

Und dennoch, für die Kategorien als reine Verstandesbegriffe will Kant mehr. Anders als bei reinen Vernunftbegriffen brauchen wir uns hier nicht mit einem rationalen Glauben zu begnügen, zu dem die metaphysische Deduktion ja schon berechtigen würde, sondern Kant meint, ihre objektive Gültigkeit unabhängig dartun zu können, eben in der transzendentalen Deduktion. Dazu aber müssten die Substantialität der Substanzen und die Kausalität der Verursachungen (und so weiter für alle Kategorien) auch noch in einer Weise epistemisch zugänglich sein, aus der sich unabhängige Belege für die rationale Setzung ihrer objektiven Gültigkeit gewinnen ließen. Rezeptiv, durch Empfindungen, sind sie aber, wie Hume gezeigt hatte, nicht epistemisch zugänglich. Also scheint guter Rat teuer.

Eine bloße Karikatur objektiver Gültigkeit läge in der Auskunft, dass die Objekte kategoriale Bestimmungen deswegen besitzen, weil wir die Kategorien in die Dinge hineinlesen, hineinprojizieren. Dann wären die Kategorien unsere subjektive Zutat zum Realen und gerade nicht objektiv gültig. Andererseits können wir sie dem Realen, siehe Hume, nicht rezeptiv entnehmen; also müssen wir sie aktiv, in diskursiver Spontaneität in die Dinge projizieren. Aber das betrifft nur unser Erkennen, nicht das objektive Sein der Dinge. Kants geniale Beweisidee in Sachen objektiver Gültigkeit der Kategorien lässt sich auf das Stichwort einer konservativen Projektion zuspitzen: Wir projizieren im Denken diejenigen kategorialen Bestimmungen auf oder in die Dinge, die ihnen an und für sich, schon unabhän- 
gig von unserem Projizieren, zukommen. Wir verändern sie nicht in unserem spontanen Projizieren, sondern konservieren sie so, wie sie als objektive Erscheinungen an ihnen selber sind.

Ein paradigmatisches Beispiel konservativer Projektion ist das Lesen von simplen Texten. Der Sinn einer schriftlichen Mitteilung lässt sich nicht rezeptiv, in visueller Wahrnehmung erkennen, sonst würde ein Tier, ein Kleinkind, ein Analphabet ihn ebenso sehen wie eine kompetente Leserin. Vielmehr muss er von der Leserin nach einer komplizierten Projektionsmethode in die Schriftzeichen hineingelesen werden, und dies, obwohl er unabhängig vom Leseakt bereits in den Schriftzeichen liegt. Die Leserin erfindet keinen Sinn zu den Buchstaben hinzu, sondern liest, was der Autor geschrieben hat. Die Sinnprojektion im Lesen ist insofern konservativ, nicht kreativ: ein Finden, kein Erfinden.

Ganz analog soll es sich mit den Kategorien verhalten. Indem wir über die Dinge reden, d. h. urteilen, projizieren wir den Logos auf sie, Hegelisch gesprochen den Begriff, der ihnen auch an und für sich schon zukommen soll. Dabei tritt der Logos in zweierlei Form auf. Sprachseitig hat er propositionale Form und differenziert sich aus in die syllogistisch relevanten Aspekte des Urteils. Weltseitig nimmt er kategoriale Form an, die der propositionalen zwar genau korrespondiert, aber nicht dasselbe ist wie sie. Wäre die kategoriale Form mit der propositionalen Form schlicht einerlei, so wären wir auf eine Tatsachenontologie festgelegt, also auf die Realität nicht nur von Partikularien, sondern auch von Universalien sowie von einem logischen Kitt, der Partikularien und Universalien so verbinden müsste, wie die Kopula im Satz das Subjekt mit dem Prädikat verbindet. (Wittgenstein hat im Tractatus eine ähnliche Position vertreten: Ein und dieselbe singuläre logische Konstante kommt bei ihm weltseitig als logische Form der Wirklichkeit und sprachseitig als logische Form der Abbildung vor.) Dem aber hat Kant bereits in der metaphysischen Deduktion vorgebeugt, als er die Aspekte der logischen Form eigens von der propositionalen in die kategoriale Tonart transponierte. Der Form des kategorischen Urteils beispielsweise entspricht weltseitig nicht die (kategorische) Tatsache, sondern die Substanz.

Wie aber kann diese Beweisidee realisiert und gezeigt werden, dass unsere Urteilspraxis eine konservative, keine kreative Projektion von kategorialer Form ist? Kants Antwort tritt im zweiten Beweisschritt der B-Deduktion hervor: Es muss gezeigt werden, dass das reine Mannigfaltige des Raumes und der Zeit schon unabhängig von möglichen empirischen Inhalten kategorial vorstrukturiert ist. Zu diesem Zweck muss man auf die unendliche Teilbarkeit von Raum und Zeit als kontinuierlichen Größen rekurrieren und zeigen, dass diese Kontinua immer schon zu infinitesimalem Staub zerfallen wären, wenn nicht der Logos als Garant ihrer Einheit und Kontinuität fungierte. Raum und Zeit verlangen also die kategoriale Form, die das weltseitige Korrelat der propositionalen Form ist. In der Folge muss dann auch alles, was in Raum und Zeit auftreten und uns sinnlich gegeben werden kann, kategoriale Form besitzen.

Diesen Raum-Zeit-Bezug von Kants Beweis hat Hegel deflationär behandelt, und so fällt seine Version der konservativen Projektion etwas weniger pointiert aus als die kantische. Der Logos oder Begriff lässt sich zwar auch nach Hegel einerseits als Paradigma einer konservativen Projektion verstehen, weil in ihm das An-und-für-sich-Sein oder die Objektivität mit dem Gesetztsein oder Projiziertsein zusammenfällt. Das An-und-für-sich-Sein ist im Begriff „unmittelbar als Gesetztseyn“, sagt Hegel, der Begriff daher „absolute Bestimmtheit“ und gleichwohl „einfache Identität“. ${ }^{3}$ In und mit dem Begriff wird also eigens gesetzt oder projiziert, was an und für sich objektiv - vorhanden ist. Aber Hegel akzentuiert die Kreativität des Begriffs, indem er darauf abhebt, dass das An-und-für-sich-Sein im Gesetztsein erst seine Vollendung erfährt. Die Projektion ist insofern nicht gänzlich konservativ.

In den Bemerkungen über den „Begriff im allgemeinen“, mit denen Hegel die Begriffslogik einleitet, rühmt er Kants transzendentale Deduktion zunächst wie folgt:

3 G. W. F. Hegel, Wissenschaft der Logik. Zweiter Band. Die subjektive Logik (1816), Gesammelte Werke, Band 12, hg. v. Friedrich Hogemann und Walter Jaeschke, Hamburg 1981 (fortan: WdL 1816), 16. 
Es gehört zu den tiefsten und richtigsten Einsichten, die sich in der Kritik der Vernunft finden, daß die Einheit, die das Wesen des Begriffs ausmacht, als die ursprünglich-synthetische Einheit der Apperception, als Einheit des: Ich denke, oder des Selbstbewußtseyns erkannt wird. - Dieser Satz macht die sogenannte transcendentale Deduction der Categorie aus [...]. ${ }^{4}$

Kant würde sagen: Er macht den Anfang einer transzendentalen Deduktion aus; ${ }^{5}$ denn es fehlt noch der zweite Beweisschritt, der Raum und Zeit als die Formen unserer sinnlichen Anschauung ins Spiel bringen muss. In der Folge fährt Hegel dann fort:

Durch das Begreiffen wird, das An-und-Fürsichseyn, das er [der Gegenstand] im Anschauen und Vorstellen hat, in ein Gesetztseyn verwandelt; Ich durchdringt ihn denkend. Wie er aber im Denken ist, so ist er erst an und für sich; [...] das Denken hebt seine Unmittelbarkeit, mit der er zunächst vor uns kommt, auf und macht so ein Gesetztseyn aus ihm; diß sein Gesetztsein aber ist sein An-und-Fürsichseyn, oder seine Objectivität. ${ }^{6}$

Offensichtlich ist das Setzen hier als kreativer konzipiert denn als ein bloßes Explizitmachen oder konservatives Projizieren dessen, was an sich schon vorhanden ist. Im Begreifen oder Denken soll nämlich das unmittelbare An-und-für-sich-Sein nicht etwa nur durch ein Gesetztsein angereichert, sondern vollends in ein Gesetztsein verwandelt werden, welches dann erst das wahre An-und-für-sich-Sein sei. Hegel denkt an den logischen Fortgang vom Sein über das Wesen zum Begriff, d. h. an den Fortgang von der Unmittelbarkeit über die Reflexion zu demjenigen An-undfür-sich-Sein, das mit dem Gesetztsein identisch geworden ist. In der Philosophie des Geistes könnte man den Fortgang auf die Formel bringen: Der Homo sapiens vollendete, als ihm die Rede zuteilwurde, das An-und-für-sich-Sein der Welt, indem er es in Gesetztsein verwandelte. Mit Kant wäre eher zu sagen: Der Homo sapiens machte die unsichtbare kategoriale Struktur der Dinge erkennbar, als er über sie zu reden begann. Aber ich will diese Differenz zwischen Hegel und Kant nicht überbetonen, zumal Kant durch meine exegetische Rede von der konservativen Projektion vielleicht in eine allzu realistische Ecke gestellt wird, die ich zwar schätze, in die er aber nicht mit allem, was er sagt, zu passen scheint, weswegen er ja bisweilen sogar als Konstitutionsidealist aufgefasst werden konnte. Statt der Frage nachzugehen, ob Kant der realistischere Philosoph ist als Hegel, möchte ich mit Überlegungen zum Setzen oder Projizieren schließen, die an Hegels Bemerkungen über die Stufen der Reflexion zu Beginn der Wesenslogik anknüpfen, um von dort her noch einmal Licht auf Kants Unternehmen einer transzendentalen Deduktion fallen zu lassen.

\section{Setzende, äußere und bestimmende Reflexion}

Der Terminus „setzen“, lateinisch „ponere“, als Gegenbegriff zu „aufheben“, „tollere“, ist für unsere Zwecke wie überhaupt in der Logik und Philosophie von zentraler Bedeutung. Einige Beispiele: Wir schließen modo ponente und modo tollente - in setzender und aufhebender Weise. Oder nehmen wir Fichtes Begriff der Tathandlung, in der das Ich sich selber setzt. Oder denken wir drittens daran, dass wir Aussagen als wahr und ipso facto Sachverhalte als bestehend oder als Tatsachen setzen und Aussagen aufheben und verneinen. Unsere allgemeine epistemische Praxis des Erhebens von Wahrheitsansprüchen ist nichts anderes als eine Praxis des Setzens von Aussageinhalten als wahr bzw. als Tatsachen.

4 WdL 1816, $17 \mathrm{f}$.

5 Vgl. KrV B 144.

6 WdL 1816, 18. 
In der Regel sind Setzungen von Aussageinhalten als objektive Setzungen intendiert, d. h., wir setzen, es sei so und so ganz unabhängig davon, dass wir dies so setzen. Darin äußert sich unsere realistische Grundhaltung, die definitorisch ist für das, was Reinhold (und mit ihm Hegel) das Bewußtsein nannte, bzw. für das, was ich soeben unsere Praxis des Erhebens von Wahrheitsansprüchen genannt habe und was bei Sellars und Brandom als das Spiel des Gebens und Forderns von Gründen firmiert. In der Reflexionslogik fasst Hegel dieses objektivierende Setzen näher als Voraussetzen und sagt, es sei ein Setzen als nicht gesetzt. Beispielsweise setze ich als nicht gesetzt, sondern als unabhängig von meinem Setzen der Fall seiend, dass wir hier und jetzt in Wien sind. Wie kann ich aber, wenn es unabhängig von meinem Setzen sich so verhalten soll, diesen Sachverhalt erkennen und wissen? Das ist die skeptische Grundfrage, die den Realismus als sein Alter ego begleitet und auf die es prima facie nur eine plausible Antwort zu geben scheint: Überhaupt nicht. Zuviel Realismus führt leicht zum Skeptizismus.

Nennen wir dieses realistische Setzen (Voraussetzen, Setzen als nicht gesetzt) das objektivierende oder objektive Setzen. Quine bekennt sich zu ihm, wenn er Menschen, Stöcke und Steine als unsere Setzungen deklariert und zugleich seinen robusten Realismus bezüglich Menschen, Stöcken und Steinen proklamiert. ${ }^{7}$ Nichts gegen den robusten Realismus; aber in ihm zeigt sich der Gegensatz des Bewußtseins oder, mit Sartre zu reden, unsere mauvaise foi, die sich durch all unser Fürwahrhalten hindurchzieht. Wir setzen etwas als objektiv der Fall seiend und erkennen mit der Unabhängigkeit des Gesetzten zugleich unsere Fehlbarkeit im Setzen an, somit das Setzen als ein bloßes Setzen, dem vielleicht nichts Objektives entspricht. Ein bloßes Setzen aber fällt bemerkenswerterweise - im Resultat mit seinem Gegenteil, dem Aufheben, zusammen. Was ich bloß setze, hat nur Gesetztsein, kein Ansichsein. Daher kann Hegel beispielsweise von der Notwendigkeit der Substanz sagen, dass sie im Begriff ,zugleich als aufgehoben, oder als Gesetztseyn ist" " ${ }^{8}$ Indem ein Sein als Gesetztsein ist, ist es ipso facto aufgehoben - so eng können ponere und tollere beieinanderliegen. Dementsprechend ist das Erheben von Wahrheitsansprüchen oder das Spiel der Gründe nur möglich mit einem geheimen Vorbehalt, einer Mentalreservation; denn jeder Glaube ist von dem Gedanken an Objektivität und Fehlbarkeit angekränkelt und ist, indem wir dies verdrängen, schlechter Glaube, mauvaise foi. Dies ist die Weise des Bewußtseins, des Spiels der Gründe, der epistemischen Endlichkeit.

Das epistemologische Gegenmodell zum bloßen Setzen ist dasjenige einer Erkenntnis durch Bekanntschaft (im Russellschen Sinn) oder Erkenntnis durch Anschauung, gleichviel ob durch sinnliche oder intellektuelle. In der Anschauung gibt es keine (bloßen) Setzungen, keine (bloßen) Ansprüche, kein Objektivieren, keine Fehlbarkeit, sondern unmittelbares Sein und sacherfülltes, irrtumsimmunes Erfassen. Eine Anschauung ist nicht zweiwertig, wahr oder falsch wie das Urteil, sondern einwertig: wahr simpliciter. Aristoteles hat in Metaphysik $\Theta 10$ diesen Sachverhalt wie folgt thematisiert. Unterhalb der Urteilszweiwertigkeit und Urteilssynthesis, sagt er, „ist das Wahre das Vernehmen [des Der-Fall-Seienden]; das Falsche gibt es [hier] nicht, auch keine Täuschung, sondern höchstens Unwissen [im Sinn von Nichtvernehmen]“.9 Dieses schlichte, unmittelbare, nicht-setzende Erkennen bar der Objektivität, Subjekt-Objekt-Differenz, Irrtumsmöglichkeit und Zweiwertigkeit ist seit alters die fixe Idee gewesen, aus welcher der Mythos des Gegebenen sich speist, eine notwendige Idee, die sich uns unabweisbar aufdrängt, aber eine unhaltbare. Hegel spielt sie über die ganze Seinslogik hinweg systematisch in ihren Höhen und Tiefen durch und macht uns zu Zeugen ihres definitiven Scheiterns im allseitigen Widerspruch der absoluten Indifferenz am Ende der Seinslogik. Deswegen muss die neue Runde des Logischen, diejenige der Wesenslogik, mit einem Kapitel über den Schein und über das Setzen - über die setzende, voraussetzende, äußere und zuletzt bestimmende Reflexion - beginnen.

7 W. V. Quine, Theories and Things, Cambridge (Mass.) und London 1981, 21.

8 WdL 1816, 15.

9 Aristoteles, Metaphysik Q 10, 1052a $1 \mathrm{f}$. 
Das Wesen ist die Sphäre der Dualität. Zwar gehört die besondere Dualität von Subjekt und Objekt noch nicht in die Logik, sondern erst in die Philosophie des Geistes. Dennoch haben wir es bereits in der Logik des Seins mit der Unmittelbarkeit der Bekanntschaft und in der Logik des Wesens dann mit der logischen Basis der Subjekt-Objekt-Dualität zu tun. Von dieser dualen Basis handelt das wesenslogische Anfangskapitel über den Schein bzw. die Reflexion und damit auch von der logischen Basis der Kritik der reinen Vernunft. Diese nämlich zehrt von der wesenslogischen Ausgangsdualität, auch wenn sie selber sich gar nicht im Logischen bewegt, sondern den Geist als Bewusstsein auffasst, wie Hegel in der Anmerkung zu § 415 der Enzyklopädie (1830) bemerkt. Hegel sagt dort:

Sie [die Kantische Philosophie] betrachtet Ich [also das Subjekt] als Beziehung auf ein Jenseitsliegendes, das in seiner abstracten Bestimmung das Ding-an-sich heißt. [...] Es ist daher für einen richtigen Sinn dieser Philosophie anzusehen, daß sie von Reinhold als eine Theorie des Bewußtseyns, unter dem Namen Vorstellungsvermögen, aufgefaßt worden ist. ${ }^{10}$

Außer als Theorie des Bewusstseins apostrophiert Hegel Kants Lehre auch als Reflexionsphilosophie oder Verstandesphilosophie, wohl eingedenk der These Jacobis, dass die Reflexion des Verstandes das Unbedingte und Absolute nicht erfassen könne. Die Reflexion steht auf der einen Seite und getrennt davon das Absolute auf der anderen, und eben dieses Verhältnis charakterisiert den Anfang der Wesenslogik.

Für die Seinslogik war es charakteristisch gewesen, dass das reine Denken in Einheit mit seinem Gegenstand fortgeschritten war und sich zuletzt im allseitigen Widerspruch der absoluten Indifferenz in eine unlösbare Inkonsistenz verstrickt hatte. Die Theorieentwicklung hatte sich bis zu dieser seinslogischen Endkatastrophe im oder am Sein selber vollzogen; das reine Denken hatte seinen Gegenstand stets in Sichtweite oder war vielmehr mit ihm identisch. Die seinslogische Endkatastrophe kann jedoch nur noch dadurch in eine weitere Theorieentwicklung umgebogen werden, dass wir den unmittelbaren Gegenstand des Denkens, also alles, was vom Sein in der absoluten Indifferenz noch übriggeblieben sein mag, zu Schein erklären. In diesem Schein ist das Denken in sich eingeschlossen und verfangen; es dringt nicht mehr durch, transzendiert nicht mehr zu seinem intendierten Gegenstand. Es tritt sich gleichsam selbst in den Weg und versperrt sich die Sicht. Statt auf ein Fenster nach draußen stößt es auf einen Spiegel, in welchem es nur sich selbst erblickt, nur sich selbst reflektiert.

Das Denken ist hier Scheinen, reine Reflexion, und in sich selbst verfangen und verstrickt. Jenseits seiner muss die Wahrheit des Seins, das Wesen, angenommen, also gesetzt werden. Dieses Annehmen, Setzen, ponere, ist ein Setzen als nicht gesetzt, ein Voraussetzen. Das Denken als Scheinen setzt sich das Wesen als seinen intendierten Gegenstand, als seine Wahrheit, voraus. Aber diese Wahrheit ist ihm (oder scheint ihm fürs erste) epistemisch unzugänglich und äußerlich. Oder vielmehr ist das Denken umgekehrt seiner vorausgesetzten Wahrheit äußerlich und vermag sie nicht zu erreichen. Die wesenslogische Theorieentwicklung bewegt sich daher anfangs ganz im Schein, obwohl sie nicht diesen, sondern das Wesen intendiert. So gehören etwa die Reflexionsbestimmungen, von denen das zweite Kapitel der Wesenslogik handelt, prima facie ganz dem Denken, nicht der Sache an. Allerdings ist bei alledem auch schon klar, dass der Schein dem Wesen nicht als ein Anderes zur Seite oder gegenüber treten kann, sondern letztlich nur das In-sich-selber-Scheinen des Wesens ist.

Zuvor, im ersten Kapitel, werden die operativen wesenslogischen Maßnahmen eingeführt (wie auch zu Beginn der Seinslogik die logische Prozessualität des Seins eingeführt wurde, nämlich in der Kategorie des Werdens und dann der Veränderung u. s. f.). Zunächst ist das Scheinen oder die Reflexion als setzende Reflexion die scheininterne Reflexion, die dann im zweiten Kapitel als Iden-

10 G. W. F. Hegel, Gesammelte Werke, Band 20, hg. v. Wolfgang Bonsiepen und Hans / Christian Lucas, Hamburg 1992, 423. 
tität, Unterschied, Widerspruch und Grund thematisiert wird. Aber sie setzt sich etwas voraus, eben das Wesen, das sie indes zunächst nicht erfassen kann. In Beziehung auf dieses Vorausgesetzte ist sie äußere Reflexion, die ihr Zielobjekt nicht erreicht. (Darin entspricht das Zielobjekt Kants Ding an sich, das durch die Setzung einer kategorialen Struktur von seiten des Subjekts nicht erreicht wird.) Es ist dies die Sphäre der Dualität, näher der Dualität von Innen und Außen, die im zweiten Abschnitt der Wesenslogik („Die Erscheinung“) untersucht wird. Doch dann zeigt sich (und zwar in abstrakter Vorwegnahme schon im ersten wesenslogischen Kapitel, konkret erst im Übergang zum dritten wesenslogischen Abschnitt), dass die Reflexion bestimmend ist. Das Vorausgesetzte bestimmt sich selbst gemäß der äußerlich auftretenden, in Wahrheit aber ihm zugehörigen und internen Reflexion. Dies ist die Thematik des dritten Abschnittes der Wesenslogik, in dem die Wirklichkeit als die Einheit des Inneren und Äußeren betrachtet wird. Nun also bekommt das Denken wieder Kontakt mit seinem intendierten Gegenstand und kann ihn wieder erfassen, wieder eins mit ihm werden.

Das reine Denken am wesenslogischen Anfang ist Schein. De facto ist es auf der Suche nach dem Wesen; aber das wissen zunächst nur wir in unserer äußeren Reflexion. Das erste Kapitel („Der Schein“) ist unsere äußere Reflexion, in der die Schicksale des Scheins und des Wesens vorweg erzählt werden. McTaggart wollte daher dieses Kapitel (wie in der enzyklopädischen Logik) entfallen lassen und mit den Reflexionsbestimmungen - d. h. mit dem zweiten Kapitel - beginnen. Dafür spricht prima facie, dass erst vom zweiten Kapitel an das reine Denken sich weiter entwickelt, indem es sich als Identität, Unterschied u. s. w. artikuliert. Aber das erste Kapitel ist doch unverzichtbar, um uns zu erklären, dass wir am Ende der Seinslogik nicht definitiv gescheitert sind, und um uns zu zeigen, wie die Entwicklung weitergehen kann. Das erste Kapitel dient, mit anderen Worten, dem Finden (und Rechtfertigen) eines neuen Anfangs.

Dabei kann Hegel die Abfolge der Reflexionsstufen vorab thematisieren, die in der Folge operativ werden, zuerst die Reflexion als bloßes Setzen, wie sie den Schein erzeugt, dann als objektivierendes Setzen oder Voraussetzen, das den gesetzten Gegenstand nicht erreicht. Sofern es den Gegenstand aber tatsächlich gibt, wird die voraussetzende Reflexion relativ zu ihm äußere Reflexion. Dies entspricht dem, was Kant das bloße Denken im Unterschied zum Erkennen nennt, wenn er sagt, dass die reine Vernunft die Seele, die Welt und Gott denken, aber nicht erkennen kann. Hingegen denkt der reine Verstand Zeitpunkte als nomologisch determiniert durch andere Zeitpunkte gemäß der Kategorie der Kausalität, und dieses Denken ist zugleich Erkennen. Hier also tritt nach Kant eine konservative Projektion in Operation bzw., wie Hegel dies nennt, eine bestimmende Reflexion. Sie ist Reflexion und als solche ihrem Gegenstand prima facie äußerlich. Aber sie soll ihn dennoch erreichen können, und ihre Bestimmungen, die sie am Gegenstand setzt, sollen zugleich seine sein, die er von sich aus mitbringt und die ihm selber in seinem An-und-für-sich-Sein angehören.

Die Begrifflichkeit, die Hegel entwickelt, um die Methode der Wesenslogik und den Übergang in den Begriff zu profilieren, ist insofern geeignet, auch Kants kritisches Programm im Kern zu beschreiben, präziser: die Leistungen und Aspekte dessen, was Kant die Spontaneität des Verstandes nennt und von der Rezeptivität der Sinnlichkeit unterscheidet. Die Spontaneität tritt als setzende, vor allem aber voraussetzende und dann in Beziehung auf das Vorausgesetzte entweder als äußere oder als bestimmende Reflexion auf. In der transzendentalen Deduktion geht Kant aus von der spontanen Synthesis, die wir im diskursiven Denken vollziehen, und zeigt, dass deren Aspekte, umgeformt in Prädikate von Objekten, im Realen schon objektiv vorhanden sind, nämlich als dessen allgemeine kategoriale Struktur. Die kategoriale Struktur lässt sich nicht rezeptiv erkennen, wie Hume gezeigt hatte; den Tieren bleibt sie also verborgen. Wir Menschen aber können und müssen sie in spontaner Synthesis ins Reale projizieren, dies jedoch in einer konservativen Projektion, die, anders als Nietzsche und Adorno befürchteten, nichts abschneidet oder hinzufügt, sondern nur dasjenige am Realen eigens setzt und zum Vorschein bringt, was an sich dort schon vorhanden ist. Darin besteht die objektive Gültigkeit der Kategorien: dass wir sie dem Realen nicht aufpfropfen, sondern dass dieses sich von selbst immer schon wie von Zauberhand gesteuert nach unseren reinen Verstandesbegriffen gerichtet hat. Hegel hat diesen 
Aspekt der kantischen Deduktion, die Pointe ihres zweiten Beweisschrittes, nicht deutlich genug gesehen, weil er ein völlig anderes philosophisches Verfahren befolgt, das ihn vom Desiderat einer transzendentalen Deduktion dispensiert und das mehr logische Struktur offenlegt als das kantische. Er mag Kant insoweit theoretisch überboten haben. Aber widerlegt oder falsifiziert ist das kantische Verfahren damit in keiner Weise.

Prof. Dr. Anton Friedrich Koch

Universität Heidelberg

Philosophisches Seminar

Schulgasse 6

D-69117 Heidelberg

a.koch@uni-heidelberg.de 